\title{
Performance Optimization of the Gasdynamic Mirror Propulsion System
}

\author{
William J. Emrich, Jr. ${ }^{1}$ and Terry Kammash ${ }^{2}$ \\ ${ }^{I}$ NASA - Marshall Space Flight Center, Bldg. 4666, Room 370,Huntsville, AL. 35812 \\ ${ }^{2}$ Dept. of Nuclear Engineering and Radiological Sciences, University of Michigan, Ann Arbor, MI 48109 \\ (256) 544-7504; bill.emrich@msfc.nasa.gov \\ (313) 764-0205; tkammash@engin.umich.edu
}

\begin{abstract}
Nuclear fusion appears to be a most promising concept for producing extremely high specific impulse rocket engines. Engines such as these would effectively open up the solar system to human exploration and would virtually eliminate launch window restrictions. A preliminary vehicle sizing and mission study was performed based on the conceptual design of a Gasdynamic Mirror (GDM) fusion propulsion system. This study indicated that the potential specific impulse for this engine is approximately $142,000 \mathrm{sec}$. with about $22,100 \mathrm{~N}$ of thrust using a deuterium-tritium fuel cycle. The engine weight inclusive of the power conversion system was optimized around an allowable engine mass of $1500 \mathrm{Mg}$ assuming advanced superconducting magnets and a Field Reversed Configuration (FRC) end plug at the mirrors. The vehicle habitat, lander, and structural weights are based on a NASA Mars mission study which assumes the use of nuclear thermal propulsion ${ }^{1}$ Several manned missions to various planets were analyzed to determine fuel requirements and launch windows. For all fusion propulsion cases studied, the fuel weight remained a minor component of the total system weight regardless of when the missions commenced. In other words, the use of fusion propulsion virtually eliminates all mission window constraints and effectively allows unlimited manned exploration of the entire solar system. It also mitigates the need to have a large space infrastructure which would be required to support the transfer of massive amounts of fuel and supplies to lower a performing spacecraft.
\end{abstract}

\section{INTRODUCTION}

One of the great deterrents to the large scale exploration of the solar system has been and continues to be the tremendous cost associated with putting the massive amounts equipment and infrastructure into space to support such endeavors. Consider, for example, the case for a manned Mars mission which by almost any account would be the easiest interplanetary mission to accomplish. If one were to use a strictly chemical system having a specific impulse $\left(\mathrm{I}_{\mathrm{sp}}\right)$ of $450 \mathrm{~s}$ to perform this mission a tremendous amount of infrastructure in the form of refueling stations and multistage expendable vehicles would be required. Nuclear thermal systems with an $\mathrm{I}_{\mathrm{sp}}$ of about $950 \mathrm{~s}$ while requiring far less infrastructure than chemical systems would, nevertheless, still require massive amounts of fuel (in the order of several times the dry weight of the transfer vehicle) to accomplish the mission. Both of these systems, while they are able to successfully accomplish a Mars mission, do so only at great cost due primarily to the massive amounts of fuel which must be transported into orbit to compensate for the inherent efficiency limitations of the engines. For more ambitious manned missions, such as to the outer planets, it is unlikely that either chemical systems or probably even nuclear thermal systems will be able to accomplish the voyage.

Ideally, for solar system exploration, one would want a vehicle with specific impulses in the range of 10,000 to $200,000 \mathrm{~s}$ and at least moderate levels of thrust. Fusion engines, if they can be built in reasonable sizes, match these requirements quite closely and would be most suitable as the primary propulsion system for an interplanetary vehicle. Such a vehicle would be quite capable accomplishing manned missions to any planet in the solar system. In the present study, one particular type of fusion engine, the Gasdynamic Mirror, was chosen as the basis for a vehicle design upon which various planetary mission analyses were performed ${ }^{2}$.

\section{ENGINE DESCRIPTION}

The Gasdynamic Mirror or GDM upon which this study is based is a modification of the simple mirror design first proposed many years ago. This particular reactor configuration for a variety of reasons seems to be particularly well suited for fusion propulsion applications. The device would operate at much higher plasma densities and with much

CP504, Space Technology and Applications International Forum-2000, edited by M. S. El-Genk

() 2000 American Institute of Physics 1-56396-919-X/00/\$17.00 
larger length to diameter ratios than previous mirror machines. Several advantages accrue from such a configuration. First, the high length to diameter ratio minimizes to a large extent certain magnetic curvature effects which lead to plasma instabilities causing a loss of plasma confinement. In particular, this feature should make it possible to suppress the magnetohydrodynamic (MHD) "flute" instability which is known to plague classical mirror fusion designs. Second, the high plasma density should result in the plasma behaving much more like a conventional fluid with a mean free path shorter than the length of the device. The short mean free path implies that a majority of the fuel ions will undergo fusion reactions prior to being reflected at the magnetic mirror reflectors at the ends of the device. This characteristic helps reduce problems associated with "loss cone" microinstabilities which result from a depletion of the particle velocity distribution function in that these instabilities will not cause the longitudinal confinement time to be reduced below a certain level.

For the present study, a number of parameters have been optimized so as to maximize the engine thrust to weight ratio subject to various constraints such as maximum permissible vehicle mass, maximum allowable magnetic field strengths, etc. The vehicle, in particular, was constrained to a maximum mass of $1500 \mathrm{Mg}$ and the mirror magnets were constrained to maximum centerline field strengths of 15 tesla. The resulting optimization yielded in the set of parameters presented in Table 1.

TABLE 1. GDM Reference Conditions.

\begin{tabular}{ll}
\hline Plasma Density $\left(\right.$ ion $\left./ \mathrm{cm}^{3}\right)$ & $2.2 \times 10^{16}$ \\
Plasma Temperature $(\mathrm{keV})$ & 10 \\
Fuel Mixture & $1 / 2 \mathrm{D}+1 / 2 \mathrm{~T}$ \\
Vacuum Beta & 0.95 \\
Gain Factor (Q) & 2.935 \\
Plasma Length (m) & 72 \\
Plasma Diameter (cm) & 8 \\
Magnetic Field at Center (tesla) & 13.8 \\
Magnetic Field at Mirror (tesla) & 15 \\
Specific Impulse (s) & 142200 \\
Thrust (N) & 22100 \\
Thrust Power (MW) & 11890 \\
Fusion Power (MW) & 14860 \\
Injector Power (MW) & 5064 \\
Bremsstralung Power (MW) & 317 \\
Synchrotron Power (MW) & 103 \\
Neutron Power (MW) & 11890 \\
Neutron Wall Load $\left(\mathrm{MW} / \mathrm{m}^{2}\right)$ & 187 \\
Specific Power $(\mathrm{kW} / \mathrm{kg})$ & 133 \\
\hline
\end{tabular}

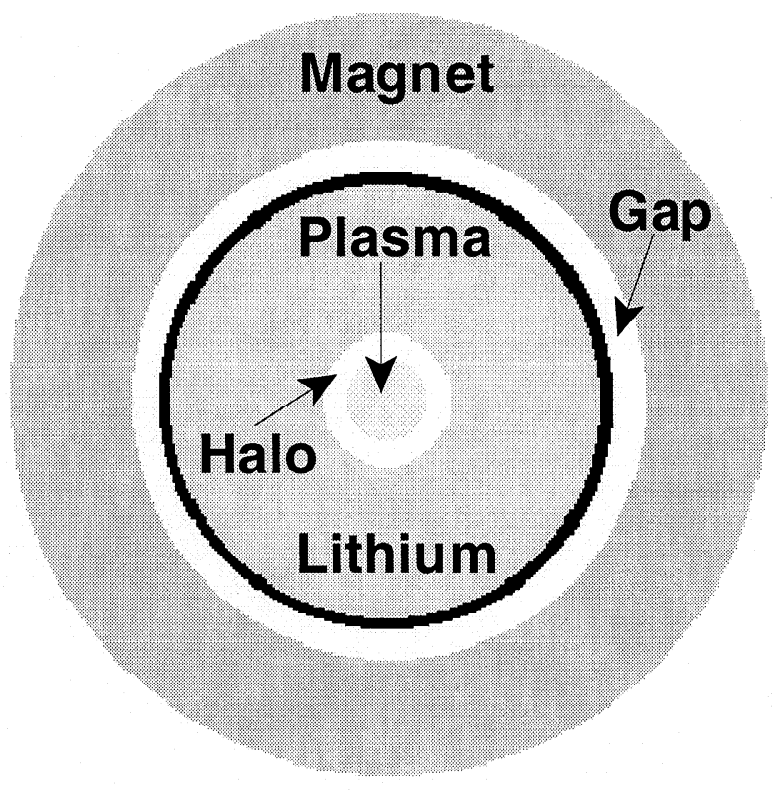

FIGURE 1. GDM Cross-sectional View.

The use of the deuterium/tritium (DT) fuel cycle for the GDM vehicle may seem a bit odd since deuterium and helium-3 are generally regarded as being preferable due to their essentially aneutronic nature.

$$
\mathrm{D}+\mathrm{T} \rightarrow{ }^{4} \mathrm{He}+\mathrm{n}(14.1 \mathrm{MeV})
$$

Unfortunately, the optimization program converged on a $\mathrm{D}$ to $\mathrm{He}^{3}$ ratio of zero. The $\mathrm{D}-\mathrm{He}^{3}$ fuel combination apparently results in vehicle lengths which are impractically long and heavy. To cope with the high flux of $14 \mathrm{MeV}$ neutrons which is the unavoidable consequence of using the DT fuel combination, therefore, it was decided to implement a design in which the first wall of the reactor is made entirely of lithium. During engine operation, the high neutron wall load resulting from the intense fusion reactions will cause the lithium to liquify. A slow rotation induced in the vehicle, will then produce centrifugal forces which will force the lithium to remain in place creating in essence a liquid first wall. A number of advantages accrue from this design. First, the material problems associated with radiation damage to the first wall are greatly reduced due to its being a liquid state. Second, the lithium can act as a coolant which when pumped through an appropriate heat exchanger can be used to heat a gaseous working fluid to power a Brayton cycle thermal electric conversion system. This energy would be used to provide electrical power for the fusion neutral beam injector system and for general station keeping. Third, as 
shown below, the lithium can act as a breeding material to produce the tritium fuel required by the engine.

$$
\begin{gathered}
\mathrm{n}+{ }^{6} \mathrm{Li} \Rightarrow \mathrm{T}+{ }^{4} \mathrm{He} \\
\mathrm{n}+{ }^{7} \mathrm{Li} \Rightarrow \mathrm{T}+{ }^{4} \mathrm{He}+\mathrm{n}^{\prime}
\end{gathered}
$$

The use of bred tritium thus eliminates the need to transport large quantities of the radioactive element into orbit. Figure 1 illustrates a cross-section of the GDM engine concept used in this study.

Perhaps the most critical component in the GDM engine is the system of magnets used to confine the plasma in a stable configuration. Magnetic fields must be generated which are of sufficient strength to contain the hot fusing plasma, while at the same time not being so strong so as to overstress the engine's structural members. Calculations were performed to determine magnet sizes and current densities required to produce the necessary fields. These calculations show that with today's technology, it is possible to attain the required field strengths for the midsection magnets. Plasma physics considerations require centerline field strengths along the engine midsection to be approximately 14 tesla. The mirror magnets are a different story, however, in that for the present design, mirror centerline field strengths of 100's of tesla would normally be required. Magnetic fields having this intensity have never been produced and represent an obstacle to constructing GDM fusion engines of reasonable size. The use of Field Reversed Configurations (FRC) are proposed as a solution to this problem by creating in effect a magnetic plug which will have the effect of retarding plasma loss rates by creating high effective mirror ratios. The mirror magnets need be only strong enough to prevent the ejection of the FRC plug. These mirror fields are estimated to be only about $10 \%$ higher than the central magnets. ${ }^{3}$. For this present study, optimizations yielded the following relationships between the magnetic field strength and the overall engine weight and thrust to weight ratio. The optimizations independently varied the mirror ratios at each end of the engine, the ratio of Larmor radius to plasma radius and the plasma temperature to yield either minimum engine mass or maximum engine thrust to weight ratio. Figures 2 through 5 illustrate the results of these studies.

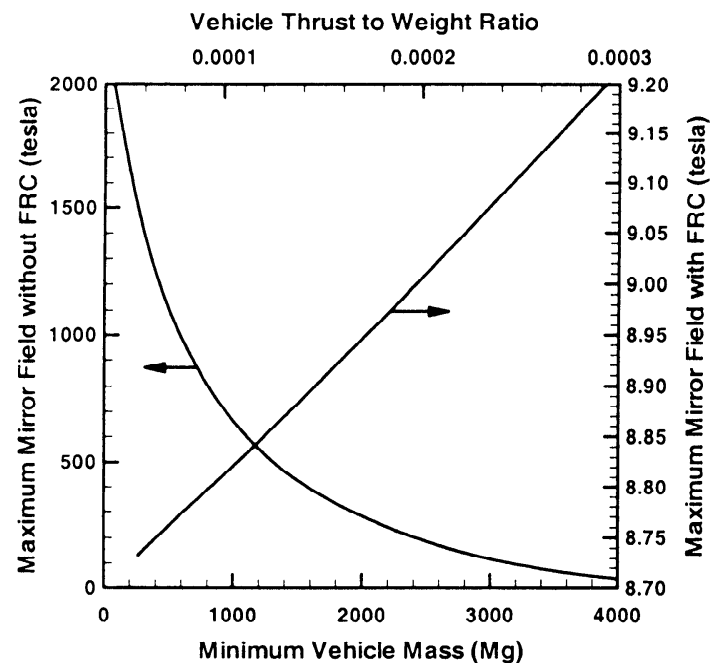

FIGURE 2. GDM Vehicle Thrust to Weight Ratio

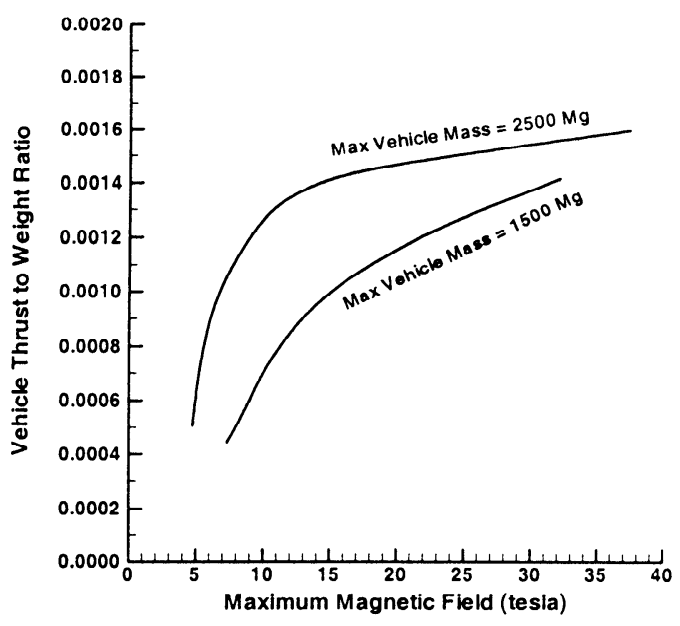

FIGURE 3. GDM Performance Characteristics

In order to maintain the fusion reactions within the engine, it is proposed that neutral beam injectors be employed both to fuel and heat the plasma. The power to run the injectors would be derived from the fusing plasma itself through a combination of direct and thermal electric energy convertors. The critical "Q" value (Energy Out / Energy In) required for the engine system to be self sustaining will, therefore, be heavily dependent on the conversion system efficiencies.

For the present study conversion efficiencies of $30 \%$ were assumed for the Brayton cycle thermal electric conversion system, $90 \%$ for the direct electric conversion system, and $100 \%$ for the neutral beam injector system. Optimization studies found that for a minimum mass engine, charged particle power should generally be split evenly between that used for thrust and that used for direct electrical energy generation. 


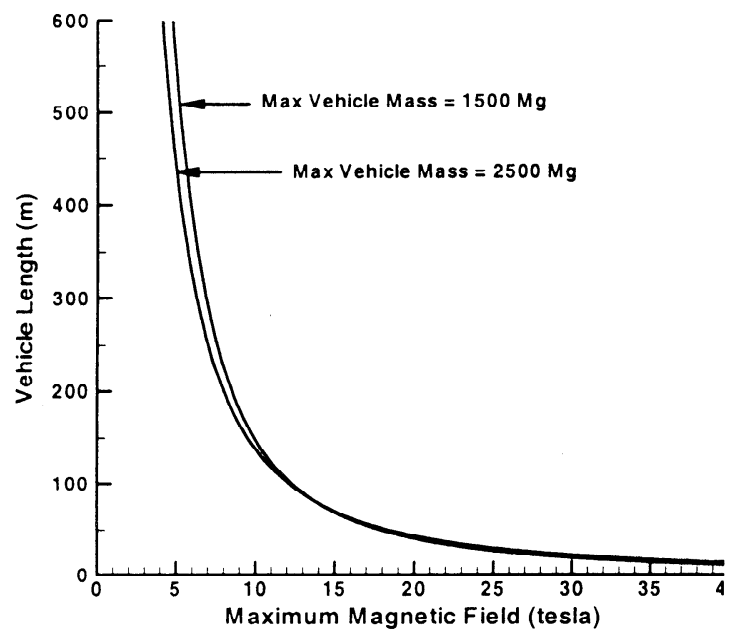

FIGURE 4. GDM Length Parametrics

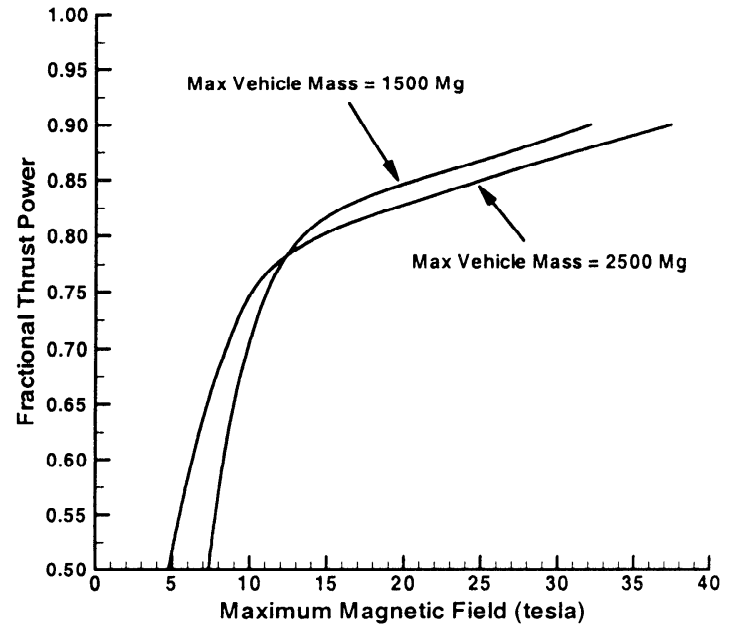

FIGURE 5. GDM Asymmetry Effects

The energy required to start the system initially comes from a capacitor bank which is charged by a set of fuel cells. The capacitor bank was sized to produce a $1000 \mathrm{MW}$-sec pulse of electricity to start the fusion reactions. Advanced capacitors currently under development are anticipated to have charge storage densities of $36 \mathrm{~kJ} / \mathrm{kg}$. The fuel cells used in the system also provide station keeping power for the vehicle when the fusion engine system is shutdown. Recharging of the fuel cells is accomplished by tapping off some of the power generated by the fusion electrical conversion systems during engine operation.

TABLE 2. GDM Vehicle Weights

\begin{tabular}{lr}
\hline Magnets (Mg) & 30 \\
Radiator (Mg) & 1077 \\
Thermal Electric Convertor (Mg) & 55 \\
Direct Electric Convertor (Mg) & 53 \\
Neutral Beam Injector (Mg) & 23 \\
Fuel Cell/Capacitor System (Mg) & 35 \\
Tritium Breeding System (Mg) & 10 \\
Lithium Shield (Mg) & 37 \\
Magnet Cooling System (Mg) & 19 \\
Structure (Mg) & 36 \\
Habitat (Mg) & 65 \\
Lander (Mg) & 60 \\
\hline Total (Mg) & 1500 \\
\hline
\end{tabular}

multiple trips to the main vehicle and to other locations on the planetary surface using locally available resources.

The habitat portion of the vehicle consists of a set of eight modified space station modules and is sized to provide long term accommodations for a crew of six. Radiation shielding is provided through the use of an internal "storm shelter" composed of foodstuffs, water, and waste products. It is estimated that approximately $100 \mathrm{kw}$ of electricity will be required to operate the vehicle, exclusive of the fusion engine. The particular design considered for this study does not include a completely closed life support system. Only the oxygen/carbon dioxide portion of the system is closed, the foodstuffs are considered consummables and must be brought along for the missions.

The lander used for this study is a modified Reusable Launch Vehicle (RLV) having a vertical takeoff, vertical landing design. This vehicle could serve as a means not only of transferring a crew and equipment to and from earth orbit but also to and from the orbits of other planetary bodies. It is anticipated that this vehicle will include a small nuclear powered in-situ fuel processing system such that it would be possible to make The system weights for all the major systems for the vehicle, including the habilat, lander, engine system, etc. were estimated and included for all the mission studies. A summary of the weights is given in Table 2 . 


\section{CONCLUSIONS}

The results of this study indicate that the fusion rocket is capable of opening the entire solar system to continuous extensive human exploration with little of the in-space infrastructure required to support the refueling operations associated with NTR or chemical systems. Voyages beyond Mars become feasible due to the ultra high efficiencies of these engines. From an economic standpoint, the benefits associated with the low mission fuel requirements characteristic of fusion systems should quickly outweigh the development cost differential perceived to be in favor of fission or chemical systems. Indeed, it may be that the development costs associated with fusion systems may be less than corresponding fission systems because of the greatly reduced test facility requirements needed to clean up the NTR hydrogen exhaust effluent. Fusion engines, contrary to popular thought, epitomize the "cheaper, faster,

better" concept to space exploration. It will only be through vehicles of this type that truly practical solar system exploration and colonization will be possible on a large scale at an affordable cost.

\section{REFERENCES}

Emrich, W. and Young, A., "Nuclear Propulsion System Options for Mars Missions," AIAA Space Programs and Technologies Conference, March 24-27, 1992, Paper \# AIAA 92-1496.

Kammash, T. and Lee, M., "Gasdynamic Fusion Propulsion System for Space Exploration," Journal of Propulsion and Power, Vol. 11, No. 3, May-June 1995, Page 544-553.

Kammash, T., Emrich, W., and Godfroy, T., "Promising Approaches to Mass Reduction of the GDM Fusion Propulsion System," $10^{\text {th }}$ NASA/JPL/MSFC/AIAA Advanced Space Propulsion Workshop, April 5-8, 1999. 